Reprod. Nutr. Dévelop., 1988, 28 Suppl. n 1, 87-88

\title{
Influence des protozoaires ciliés du rumen sur la dégradation in sacco de la paille de blé et de la luzerne
}

\author{
C. KAYOULI $\left({ }^{1}\right)$, K. USHIDA $\left({ }^{2}\right)$, S. DE SMET $\left({ }^{3}\right)$, J. P. JOUANY $\left({ }^{*}\right)$, D. I. DEMEYER $\left({ }^{3}\right)$
}

( ${ }^{*}$ Station de Recherches sur la Nutrition des Herbivores. Unité de la Digestion Microbienne, I.N.R.A., Theix 63122 Ceyrat, France.

(1) IN.A.T. - Chaire de Zootechnie, 43, av. Charles-Nicolle, 1002 Tunis - Belvédère.

(2) Kyoto Prefectoral University - Shimogamo, Kyoto 606, Japon.

(3) Research Center for Nutrition, Animal Husbandry and Meat Technology. State University of Ghent, Proefhoevestraat, 109230 Mel/e, Belgique.

Summary. Type B protozoa had a direct positive effect on the degradation rate of straw and lucerne dry matter measured by the nylon bag technique. They compensated for the decrease in bacterial activity in faunated animals, but also were more efficient than bacteria in defaunated animals. The addition of starch to a treated straw diet did not modify this situation but it lowered the degradation rate of straw and greatly increased that of lucerne.

L'objet de ce travail est de comparer la cinétique de dégradation en sachets d'une paille de blé et d'une luzerne chez des moutons faunés ou défaunés (sans protozoaires) en utilisant des sachets dont la taille des mailles permet l'entrée, soit de l'ensemble des microorganismes $(100 \mu \mathrm{m})$ soit, en théorie, des seules bactéries $(5 \mu \mathrm{m})$. Nous avons également mesuré la réaction des microorganismes à l'addition d'une source de glucides fermentescibles (amidon) à une ration peu digestible, ce paramètre semblant être important pour expliquer les divergences entre auteurs (Cf. revue de Jouany, Demeyer et Grain, 1988).

Matériel et méthodes. Six moutons mâles castrés porteurs d'une fistule permanente du rumen ont été répartis en 2 lots de 3 . Leur rumen a été défauné selon la méthode de Jouany et Senaud (1979) au cours de la première période, puis ensemencé avec un mélange de protozoaires de type B (Entodinium spp. + Eudiplodinium spp. + Eremoplastron spp. + Epidinium spp. + Isotricha spp.) pendant la deuxième période. Chaque période, qui a duré environ 10 semaines, a été divisée en deux sous-périodes pendant lesquelles un lot a reçu quotidiennement et en continu (12 repas $/ 24 \mathrm{~h}$ ) $900 \mathrm{~g}$ de paille de blé traitée par $\mathrm{NH}_{3}$ anhydre ( $3 \%), 60 \mathrm{~g}$ de farine de poisson, $30 \mathrm{~g}$ d'un complément minéral vitaminique et $15 \mathrm{~g}$ de $\left(\mathrm{NH}_{4}\right)_{2} \mathrm{SO}_{4}$. Dans l'autre lot, $180 \mathrm{~g}$ de paille de blé traitée ont été remplacés par le même poids de mais grain broyé et aggloméré. Les régimes ont été permutés pour la $2^{\mathrm{e}}$ sous-période.

Des sachets réalisés en nylon calibré (mailles de 100 et $5 \mu \mathrm{m}$ ) contenant $3 \mathrm{~g}$ de paille de blé ou de luzerne $\left(2^{\mathrm{e}}\right.$ cycle, séchée artificiellement), sous forme broyée (grille de $4 \mathrm{~mm}$ ) ont séjourné pendant différents temps $(12,24,48,72 \mathrm{~h}$ pour la paille ; $10,24,48 \mathrm{~h}$ pour la luzerne) dans le rumen ( 2 sachets par durée

(*) Adresse pour les demandes de tirés-à-part. 
et par mouton). Une analyse de variance a été effectuée pour chaque durée afin de caractériser l'effet "protozoaires», et l'effet «maïs» sur la disparition de la matière sèche (MS) corrigée, pour les sachets de $100 \mu \mathrm{m}$, des pertes physiques estimées par la diminution du poids des sachets contenant les substrats après lavage ( 3 fois $5 \mathrm{~min}$ ), en machine, à l'eau froide.

Résultats (tabl. 1). A l'exception du point $48 \mathrm{~h}$, la présence de protozoaires ciliés dans le rumen a toujours significativement amélioré la dégradation de la MS de la paille de blé en sachets de $100 \mu \mathrm{m}$. Cette dégradation n'évolue plus au-delà de $48 \mathrm{~h}$ dans les rumens défaunés alors qu'elle augmente jusqu'au temps $72 \mathrm{~h}$ dans les rumens faunés. L'effet positif des protozoaires sur la dégradation de la MS de la luzerne n'apparaît que pendant les premières $24 \mathrm{~h}$ pour les 2 régimes étudiés.

TABL. 1. - Cinétique du taux de disparition de la matière sèche (\% de l'introduit) de paille de blé et de la luzerne, en sachets de 100 et $5 \mu$ (valeurs entre parenthèses pour $5 \mu \mathrm{m}$

\begin{tabular}{|c|c|c|c|c|c|c|c|c|}
\hline \multirow[b]{2}{*}{ Règime } & \multicolumn{5}{|c|}{ Paille } & \multicolumn{3}{|c|}{ Luzerne } \\
\hline & animaux & $12 \mathrm{~h}$ & $24 h$ & $48 h$ & $72 \mathrm{~h}$ & $10 \mathrm{~h}$ & $24 \mathrm{~h}$ & $48 h$ \\
\hline \multirow{2}{*}{ Paille $\mathrm{NH}_{3}$} & défaunés & 14,8 & $23,1 \quad(19,8)$ & $34,0(18,4)$ & 35,0 & $23,7(23,7)$ & $44,8(29,0)$ & 56,0 \\
\hline & faunés & 18,0 & $26,0(13,1)$ & $36,2(19,8)$ & 39,3 & $33,0(22,2)$ & $51,2(26,6)$ & 56,1 \\
\hline \multirow{2}{*}{$\begin{array}{l}\text { Paille } \mathrm{NH}_{3} \\
+ \text { maïs }\end{array}$} & défaunés & 12,5 & $21,6(15,6)$ & $33,3(15,8)$ & 33,8 & $32,3(25,4)$ & $47,3(28,5)$ & 57,0 \\
\hline & faunés & 16,6 & $24,0(13,6)$ & $33,2(19,1)$ & 37,0 & $38,9(20,2)$ & $50,2(25,1)$ & 56,7 \\
\hline \multirow{2}{*}{\multicolumn{2}{|c|}{$\begin{array}{l}\text { Effet «protozoaires» } \\
\text { Effet «mais } ~\end{array}$}} & $* * *$ & $*^{* *}\left({ }^{* * *}\right)$ & $N S\left(^{*}\right)$ & $* *$ & $* * *\left({ }^{* * *}\right)$ & $* *\left({ }^{* *}\right)$ & NS \\
\hline & & ** & ${ }^{* *}\left({ }^{*}\right)$ & $N S(N S)$ & NS & $* *$ (NS) & $N S(N S)$ & NS \\
\hline
\end{tabular}

NS $=$ différence non significative $;{ }^{*} \mathrm{P}<0,05 ;{ }^{* *} \mathrm{P}<0,01 ;{ }^{* * *} \mathrm{P}<0,001$

L'addition de maïs à la ration de base entraîne une diminution de la dégradation de la MS de la paille de blé pendant les 24 premières heures qui est quantitativement égale dans les rumens faunés et défaunés. La dégradation de la MS de la luzerne est fortement augmentée pendant les 10 premières heures par la présence de maîs dans la ration des animaux. L'effet «protozoaires " n'a pas été modifié par l'apport de ce complément énergétique.

La plus faible dégradation de la MS de paille et de luzerne en sachets à pores de $5 \mu \mathrm{m}$ est associée à la présence de bactéries de taille réduite et homogène, ainsi qu'à l'absence de champignons. Ces premiers résultats d'observations microscopiques montrent que, bien qu'importante en nombre, cette flore simplifiée a une activité hydrolytique plus faible que celle de la population bactérienne totale. $\mathrm{Ce}$ fait est confirmé par la caractérisation de la dégradation des tissus. En outre, la rétention à l'intérieur des sachets à pores de $5 \mu \mathrm{m}$ des particules de petite taille formées au cours de la dégradation microbienne, accentue les écarts d'activité microbienne entre les 2 types de sachets utilisés.

Jouany J. P., Senaud J., 1979. Ann. Biol. anim. Bioch. Biophys., 19, 619-624.

Jouany J. P., Demeyer D. I., Grain J., 1988. Anim. Feed Sci. Technol. 21, 229-265. 